\title{
Field theoretic study of a cold Fermi gas in the unitary limit
}

\section{Matthew Wingate}

Institute for Nuclear Theory, University of Washington, Seattle, WA 98195-1550, USA *

E-mail: M.Wingateddamtp.cam.ac.uk

\begin{abstract}
Trapped and cooled gases of alkali atoms can be manipulated to exhibit a variety of interesting phenomena. For example, dilute gases of fermionic atoms, in 2 hyperfine states, can be cooled to temperatures where they become superfluid. An external field can be applied to tune the scattering length $a$. When $|a|$ exceeds the interparticle spacing, nonperturbative tools are needed to study the system theoretically. The unitary limit, $|a| \rightarrow \infty$, is particularly interesting due to its universality and symmetry. Lattice field theory and effective field theory can be used to systematically calculate properties of this system. Results are presented for the finite temperature phase transition and for behavior near zero temperature.
\end{abstract}

XXIVth International Symposium on Lattice Field Theory

July 23-28, 2006

Tucson, Arizona, USA

*address after 1 Sep 2006: DAMTP, Wilberforce Road, University of Cambridge, Cambridge CB3 0WA, UK. 


\section{Introduction}

In this talk I present results from studying a dilute gas of 2 species of nonrelativistic fermions interacting through a short range attraction. The most relevant experimental realization of this system is the trapping and cooling of fermionic isotopes of alkali atoms, a vibrant activity in many labs around the world. These atomic gas experiments, and the theory which describes them, can also be considered to be models of a dilute neutron gas.

The Hamiltonian is the sum of kinetic energy operators and a short range potential

$$
H=-\frac{1}{2}\left(\sum_{i=1}^{N_{1}} \nabla_{i}^{2}+\sum_{j=1}^{N_{2}} \nabla_{j}^{2}\right)+\sum_{i=1}^{N_{1}} \sum_{j=1}^{N_{2}} v\left(\left|\mathbf{r}_{i}-\mathbf{r}_{j}\right|\right) .
$$

This work is concerned with equal populations of the 2 species, $N_{1}=N_{2} \equiv N$. We adopt nonrelativistic units $\hbar=m=1$ since the fermion mass $m$, not the speed of light, is the relevant kinematic factor relating temporal and spatial units. Realistic potentials are the van der Waals potential $v(r) \sim\left(r_{0} / r\right)^{6}$ for atomic gases and the Yukawa potential $v(r) \sim \exp \left(-r / r_{0}\right) / r$ for a neutron gas. In either case $r_{0}$ is the length scale characteristic of the specific potential.

As we know, low momentum processes do not probe short distances, so under the right conditions, the many body physics is the same for atoms and neutrons. The typical momentum in a fermion gas is the Fermi momentum, defined through the particle density $n=N / V$ to be $k_{F} \equiv\left(3 \pi^{2} n\right)^{1 / 3}$. Quantitatively then, the details of the potential do not affect the physics when $k_{F} r_{0} \ll 1$, which we call the dilute regime. The atomic gases are indeed dilute; interparticle distances are typically 2-3 orders of magnitude larger than $r_{0}$. Although realistic neutron matter is never in the dilute regime - without high densities of about $0.1 \mathrm{GeV}$ per $(\mathrm{fm})^{3}$, neutrons $\beta$-decay to protons - idealized dilute neutron matter is a valid theoretical limit of realistic neutron matter and a warm-up for nuclear matter.

Looking at an effective range expansion of the scattering amplitude,

$$
\mathscr{A}=\frac{1}{-1 / a+\frac{1}{2} k^{2} r_{0}+\ldots-i k}
$$

(defined through the $S$ matrix as $S \equiv 1+i k \mathscr{A}$ ) we can see that the only relevant parameter is the S-wave scattering length $a$ in the dilute regime. (Higher partial waves are negligible at low energies.) Note we follow the convention is that $a<0$ corresponds to the 2-body system having no bound state. With $-1 \ll k_{F} a<0$ the ground state is a Bardeen-Cooper-Schrieffer superfluid, while at $0<k_{F} a \ll 1$ tightly bound difermion molecules are the ground state and form a Bose-Einstein condensate at low temperatures. Mean field theory provides an accurate description in both of these extremes. The case of particular interest to us is $k_{F}|a| \gg 1$, which happens when a bound state is close to threshold. Here fluctuations dominate and mean field theory is of no use. In particular, when $|a| \rightarrow \infty$, the scattering length is no longer a physical length scale. The only length scale left in the problem is $k_{F}^{-1}$ (or the interparticle spacing $n^{-1 / 3}$ ), giving universal physics. This is called the unitary regime.

The atomic physics experiments are pristine, versatile environments to study the dependence of many body physics on scattering length. The 2 species of fermions in the traps are 2 hyperfine 
spin states, which react differently to an external magnetic field. By tuning this magnetic field to a Feshbach resonance, the scattering length can be fine-tuned to infinity.

Large scattering lengths arise in nuclear physics as well. Low energy nucleon-nucleon scattering is governed by one-pion exchange, so dimensional analysis would suggest that the scattering length should be the same size as the pion Compton wavelength, $1.4 \mathrm{fm}$. However, in fact the $n n$ scattering length is $-18.5 \mathrm{fm}$, and in $n p$-scattering $a_{s}=-23.76 \mathrm{fm}$ and $a_{t}=5.42 \mathrm{fm}$. Thus nature exhibits an order-of-magnitude of fine tuning. Direct Monte Carlo calculation from lattice QCD of $N N$ scattering and binding as a function of quark mass is underway and should pin down how this fine tuning arises from QCD. The study of the unitary Fermi gas is relevant for nuclear physics in the dilute, $|a| \rightarrow \infty$, and $m_{W} \rightarrow \infty$ limits.

Recent experiments have cooled atomic gases in the unitary regime to temperatures near the superfluid transition [1, 曰, 3, 田, 印. For example, striking evidence of quantized vortices signal superfluidity in a rotating gas of lithium-6 [6]. Here we discuss the application of techniques familiar to us at this conference toward this interesting system.

\section{Lattice field theory at $\boldsymbol{T}_{c}$}

Since the details of the realistic potential in (1.1) are irrelevant for dilute gases, we can replace it by a local 4-fermion interaction with a tunable bare coefficient $C_{0}$. Then the discretized Lagrangian is the same as in the attractive 3-dimensional Hubbard model

$$
\mathscr{L}=\psi^{\dagger}\left(\partial_{t}-\frac{1}{2 \xi} \nabla^{2}-\mu\right) \psi+\frac{C_{0}}{2}\left(\psi^{\dagger} \psi\right)^{2} .
$$

An attractive interaction corresponds to $C_{0}<0$. We have introduced an anisotropy relating spatial and temporal lattice spacings: $\xi \equiv b^{2} / b_{t}$. An external source for pairing can be included in the Lagrangian: $J \psi \psi+$ h.c. Doing so allows one to compute the condensate $\Sigma \equiv\langle\psi \psi\rangle$ in finite volume. The result in the limit of $V \rightarrow \infty, J \rightarrow 0$ is an order parameter for the spontaneous breaking of fermion number. Although the infinite volume limit must be taken before $J \rightarrow 0$ limit, in this work we assume $J$ is not small enough to see finite volume effects.

In order to integrate out the fermion degrees-of-freedom, an auxiliary scalar field $\phi$ is introduced to complete the square

$$
\frac{C_{0}}{2}\left(\psi^{\dagger} \psi\right)^{2} \rightarrow-\frac{1}{2 C_{0}} \phi^{2}-\phi \psi^{\dagger} \psi
$$

The result is a partition function which can be expressed as a path integral with a nonnegative integrand:

$$
Z=\int \mathscr{D} \phi \operatorname{det}\left[|J|^{2}+\tilde{K}^{\dagger} \tilde{K}\right] e^{-S_{\phi}} .
$$

It has always been known that the attractive Hubbard model has no sign problem, but interest lay in the repulsive version as a model of superconductivity in electron systems. For dilute Fermi gases, this theory provides a complete effective field theory with a continuum limit [7].

The 4-fermion coupling $C_{0}$ can be tuned to obtain any scattering length $a$ through

$$
\frac{1}{C_{0} \xi}=\frac{b}{4 \pi a}-\int_{\mathrm{BZ}} \frac{d^{3} p}{(2 \pi)^{3}} \frac{1}{|\hat{\mathbf{p}}|^{2}\left(1+|\hat{\mathbf{p}}|^{2} / 4 \xi\right)}
$$




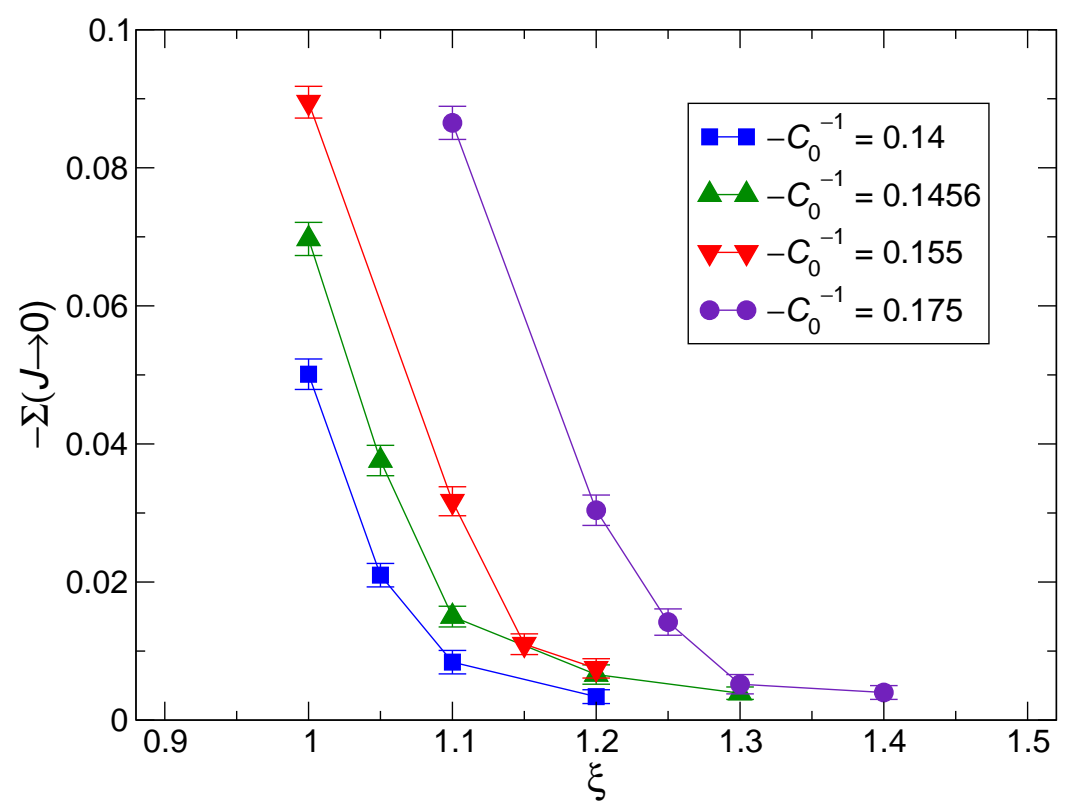

Figure 1: The order parameter, after extrapolating $J \rightarrow 0$, as a function of anisotropy for 4 values of the coupling. A rapid change in $\Sigma$ is apparent between broken (superfluid) and restored (normal) phases.

where $\hat{p}_{j}=(2 / b) \sin \left(p_{j} b / 2\right)$. The matching is obtained by requiring $2 \rightarrow 2$ scattering calculated in the vacuum from (2.1) to give the scattering amplitude (1.2).

A year ago I performed an exploratory Monte Carlo calculation of the critical temperature separating the superfluid and normal phases [8]. The lattice volume and chemical potential were fixed to $8^{3} \times 16$ and $\mu b^{2} / \xi=0.4$ respectively. As a result the fermion number density was approximately 1 fermion per 4-5 spatial lattice sites. Clearly more dilute calculations are desirable to take the continuum limit. Nevertheless, after extrapolating the external pairing source $J \rightarrow 0$, a clear jump in the pairing condensate is observed as a function of anisotropy, or temperature. Figure 1 shows the condensate vanishing to zero across the transition as the $\mathrm{U}(1)$ fermion number symmetry is restored. The four different curves correspond to four values of the bare coupling. Since the matching condition (2.4) also includes the anisotropy $\xi$, the physical scattering length is varying in the critical region, broadened out due to finite volume effects. In order to work at fixed scattering length it may be more convenient to hold both $\xi$ and $C_{0}$ fixed and vary $\mu$ across the transition. A back-of-the-envelope conversion of the critical region observed in Fig. 1 into a critical temperature yields $T_{c} / T_{F} \approx 0.04$ around $1 /|a|<0.5$.

In previous work, the attractive 3D Hubbard model has been studied in the condensed matter literature [9], and similar numerical studies have attempted to model nuclear matter [10].

An advance was made recently in Monte Carlo algorithms for this system [11, 12]. Diagrammatic Monte Carlo utilizes the convergence of the perturbative expansion for any value of the coupling, using importance sampling to generate configurations of vertices and propagators in position space. The worm updating algorithm in particular leads to a shortening of autocorrelation times [13]. Calculations have been done using a finite scaling analysis and have taken the continuum limit. They find $T_{c} / T_{F}=0.152(7)[14$, 12]. Note that at finite lattice spacings they find much 
lower values for $T_{c}$, in rough agreement with my exploratory calculation. The extrapolation to the continuum limit relies on correctly fitting the sizable discretization effects.

A calculation using different Monte Carlo methods quotes $T_{c} / T_{F}=0.23(2)$ [15], while an upper bound $T_{c} / T_{F}<0.14$ was placed in another work [16]. The systematic errors appear under tightest control in the work of Burovski et al., but discrepancy between the various methods needs to be understood and resolved.

\section{Effective field theory at $T=0$}

(This section presents collaborative work undertaken with D. T. Son [17].)

Now let us consider temperatures far below the critical temperature, to where the only relevant degrees of freedom are the phonons, fluctuations $\varphi(t, \mathbf{x})$ in the phase of the zero temperature condensate $\langle\psi \psi\rangle=|\langle\psi \psi\rangle| \exp (-2 i \varphi)$. Phonons dominate the physics when the thermal wavelength is much larger than the coherence length, roughly the size of the correlated fermion pair: $\sqrt{2 \pi / T} \gg v_{F} / \Delta_{0}$, where the Fermi velocity $v_{F}$ is defined in terms of the fermion number density and $\Delta_{0}$ is the gap in the fermion spectrum. The phonon is a massless Goldstone boson resulting from the spontaneous breaking of the $\mathrm{U}(1)$ particle number symmetry: $\psi \rightarrow \exp (i \alpha) \psi$, $\psi^{\dagger} \rightarrow \psi^{\dagger} \exp (-i \alpha)$. Below we construct an effective field theory for the phonons in a unitary Fermi gas.

In using symmetries to constrain terms in the phonon Lagrangian, it is convenient to absorb the chemical potential into a field $\theta(t, \mathbf{x}) \equiv \mu t-\varphi(t, \mathbf{x})$. The lowest-order phonon Lagrangian is

$$
\mathscr{L}_{0}=c_{0}\left(\partial_{t} \theta-\frac{|\nabla \theta|^{2}}{2}\right)^{5 / 2} .
$$

Galilean invariance forces the first derivatives of $\theta$ to appear in the linear combination $X \equiv \partial_{t} \theta-$ $|\nabla \theta|^{2} / 2$. Scale invariance dictates the functional form of $\mathscr{L}_{0}$ at the unitary point $1 / a=0$; dimensional analysis permits other powers of $X$ when $a$ is finite. The coefficient $c_{0}$ must be determined from experiment or from calculation in the microscopic theory, for example from the energy per fermion in the ground state. Below we write $c_{0}=2^{5 / 2} /\left(15 \pi^{2} \xi^{3 / 2}\right)$, where $\xi$ is the ratio of the energy per particle compared to the ideal Fermi gas energy per particle.

The lowest-order Lagrangian (3.1) is equivalent to Landau's superfluid hydrodynamics. A definite power counting scheme is needed to go beyond leading order. Since $\theta$ never appears undifferentiated, we start by counting all first derivatives of $\theta$ as terms of $\mathscr{O}(1)$. Each further derivative brings in a power of momentum $\partial_{t}^{n} \sim \partial_{i}^{n} \sim \mathscr{O}\left(p^{n}\right)$.

It is convenient to gauge the $\mathrm{U}(1)$, introducing an external field $\left(A_{0}, \mathbf{A}\right)$. We also write the interaction using an auxiliary scalar field $\sigma$ with 2 free parameters $q_{0}$ and $r_{0}$ which can be tuned to obtain any scattering length, $1 / a=0$ in particular. The microscopic Lagrangian from which we start is as follows:

$$
\begin{aligned}
\mathscr{L}= & \frac{i}{2} \psi^{\dagger} \stackrel{\leftrightarrow}{\partial}_{t} \psi-A_{0} \psi^{\dagger} \psi-\frac{g^{i j}}{2 m}\left(\partial_{i} \psi^{\dagger}-i A_{i} \psi^{\dagger}\right)\left(\partial_{j} \psi+i A_{j} \psi\right) \\
& +q_{0} \psi^{\dagger} \psi \sigma-\frac{1}{2} g^{i j} \partial_{i} \sigma \partial_{j} \sigma-\frac{\sigma^{2}}{2 r_{0}^{2}}
\end{aligned}
$$


Note that $\mathscr{L}$ is written with a nontrivial spatial metric $g_{i j}$. These external fields, $A_{0}, A_{i}, g_{i j}$, are useful tools for analyzing the system's symmetry, taking the limit where $A_{i} \rightarrow 0$ and $g_{i j} \rightarrow \delta_{i j}$ to obtain physical results. We may wish to keep $A_{0}$ nonzero to include effects of the atomic trap. $A_{0}$ always appears in combination with the chemical potential, so that $\mu-A_{0}(\mathbf{x})$ is a local effective chemical potential, e.g.

$$
X=\mu-A_{0}(\mathbf{x})-\partial_{t} \varphi-\frac{1}{2}\left(D_{i} \varphi\right)^{2} .
$$

The microscopic action $\int d t d \mathbf{x} \sqrt{g} \mathscr{L}$ is invariant under nonrelativistic general coordinate and conformal transformations. The low energy effective action must be similarly invariant. The resulting next-to-leading order Lagrangian, after taking $A_{i} \rightarrow 0$ and $g_{i j} \rightarrow \delta_{i j}$, is [17]

$$
\mathscr{L}_{2}=c_{1} \frac{(\nabla X)^{2}}{\sqrt{X}}+c_{2} \sqrt{X}\left[\left(\nabla^{2} \varphi\right)^{2}-9 \nabla^{2} A_{0}\right]
$$

Summarizing results obtained with the NLO superfluid Lagrangian, we find the leading correction to a linear phonon dispersion relation

$$
\omega=\sqrt{\frac{\xi}{3}} v_{\mathrm{F}} q\left[1-\pi^{2} \sqrt{2 \xi}\left(c_{1}+\frac{3}{2} c_{2}\right) \frac{q^{2}}{k_{\mathrm{F}}^{2}}\right]+\mathscr{O}\left(q^{5} \ln q\right) .
$$

The static density response function is found to be

$$
\chi(q)=-\frac{k_{\mathrm{F}}}{\pi^{2} \xi}\left[1+2 \pi^{2} \sqrt{2 \xi}\left(c_{1}-\frac{9}{2} c_{2}\right) \frac{q^{2}}{k_{\mathrm{F}}^{2}}\right]+\mathscr{O}\left(q^{4} \ln q\right) .
$$

And the static transverse response function is

$$
\chi^{T}(q)=-9 c_{2} \sqrt{\frac{\xi}{2}} k_{\mathrm{F}} q^{2}+\mathscr{O}\left(q^{4} \ln q\right) .
$$

A dispersion relation requires that $\chi^{T}(q)<0$; consequently $c_{2}$ must be positive. The predictivity of the effective field theory is apparent since the 3 observables over-constrain the 2 free parameters. Present experiments are not able to go to low enough temperatures to test these predictions. However, Monte Carlo calculations should be able to bridge the gap between low temperatures, where phonons govern the physics, to temperatures around $T_{c}$, where experiments can be performed. Perhaps these calculations will be able to test the predictions of this effective theory.

The alert reader might have noticed that the logarithmic corrections due to loop diagrams are suppressed compared to the NLO terms by another power of $p^{2}$, in contradistinction to the chiral logarithms. In chiral perturbation theory, the logarithms appearing at NLO arise from interaction terms where the pion field appears undifferentiated, e.g. $\left(\boldsymbol{\pi} \cdot \partial_{j} \boldsymbol{\pi}\right)\left(\boldsymbol{\pi} \cdot \partial^{j} \boldsymbol{\pi}\right)$. Such terms are not allowed in the (abelian) U(1) effective theory.

Let us close by emphasizing a few features of this work. It is remarkable that imposing nonrelativistic general coordinate invariance imposes additional constraints on the phonon Lagrangian beyond those dictated by Galilean invariance. For example, Galilean invariance would relax a linear combination of operators dictated by general coordinate invariance, so that $\chi^{T}(q)$ would depend on 3, not 2, low energy constants. This does not happen with relativistic theories: any Lorentz invariant term may be made general coordinate invariant quite simply. However, the nonrelativistic transformation is just the $c \rightarrow \infty$ limit of the relativistic transformation. 
One might question the physical justification of requiring the fermion Lagrangian (3.2) to have this coordinate invariance - after all, the microscopic degrees-of-freedom are atoms whose phenomenologically successful potentials are not general coordinate invariant. However, we take it as a valid first principle that, since the underlying dynamics in atoms are described by QED and QCD, which are general coordinate invariant theories, the nonrelativistic remnant of this invariance persists and has predictive, refutable consequences as discussed above.

Similarly, it is a logical possibility that the conformal invariance we impose need not be realized by nature. (Then $\left(\nabla^{2} \varphi\right)^{2}$ and $\nabla^{2} A_{0}$ would be linearly independent in (3.4).) However, the fact that the free Schrödinger equation is scale and conformally invariant [18] hints that the unitary Fermi gas should also possess the same symmetry.

\section{Acknowledgments}

This work was supported by DOE grant number DE-FG02-00ER41132.

\section{References}

[1] M. Bartenstein, A. Altmeyer, S. Riedl, S. Jochim, C. Chin, J. H. Denschlag, and R. Grimm, Phys. Rev. Lett. 92, 120401 (2004).

[2] C. A. Regal, M. Greiner, and D. S. Jin, Phys. Rev. Lett. 92, 040403 (2004).

[3] M. W. Zwierlein, J. R. Abo-Shaeer, A. Schirotzek, C. H. Schunck, and W. Ketterle, Phys. Rev. Lett. 92, 120403 (2004).

[4] J. Kinast, S. L. Hemmer, M. E. Gehm, A. Turlapov, and J. E. Thomas, Phys. Rev. Lett. 92, 150402 (2004).

[5] T. Bourdel, L. Khaykovich, J. Cubizolles, J. Zhang, F. Chevy, M. Teichmann, L. Tarruell, S. J. J. M. F. Kokkelmans, and C. Salomon, Phys. Rev. Lett. 93, 050401 (2004).

[6] M. W. Zwierlein, J. R. Abo-Shaeer, A. Schirotzek, C. H. Schunck, and W. Ketterle, Nature 435, 1047 (2005), cond-mat/0505635.

[7] J.-W. Chen and D. B. Kaplan, Phys. Rev. Lett. 92, 257002 (2004).

[8] M. Wingate (2005), cond-mat/0502372.

[9] A. Sewer, X. Zotos, and H. Beck, Phys. Rev. B 66, 140504(R) (2002).

[10] H. M. Müller, S. E. Koonin, R. Seki, and U. van Kolck, Phys. Rev. C61, 044320 (2000).

[11] A. N. Rubtsov, V. V. Savkin, and A. I. Lichtenstein (2004), cond-mat/0411344.

[12] E. Burovski, N. Prokof'ev, B. Svistunov, and M. Troyer, New J. Phys. 8, 153 (2006), cond-mat/0605350.

[13] N. Prokof'ev and B. Svistunov, Phys. Rev. Lett. 87, 160601 (2001).

[14] E. Burovski, N. Prokof'ev, B. Svistunov, and M. Troyer, Phys. Rev. Lett. 96, 160402 (2006).

[15] A. Bulgac, J. E. Drut, and P. Magierski, Phys. Rev. Lett. 96, 090404 (2006).

[16] D. Lee and T. Schäfer, Phys. Rev. C 73, 015202 (2006).

[17] D. T. Son and M. Wingate, Annals Phys. 321, 197 (2006), cond-mat/0509786.

[18] C. R. Hagen, Phys. Rev. D5, 377 (1972). 